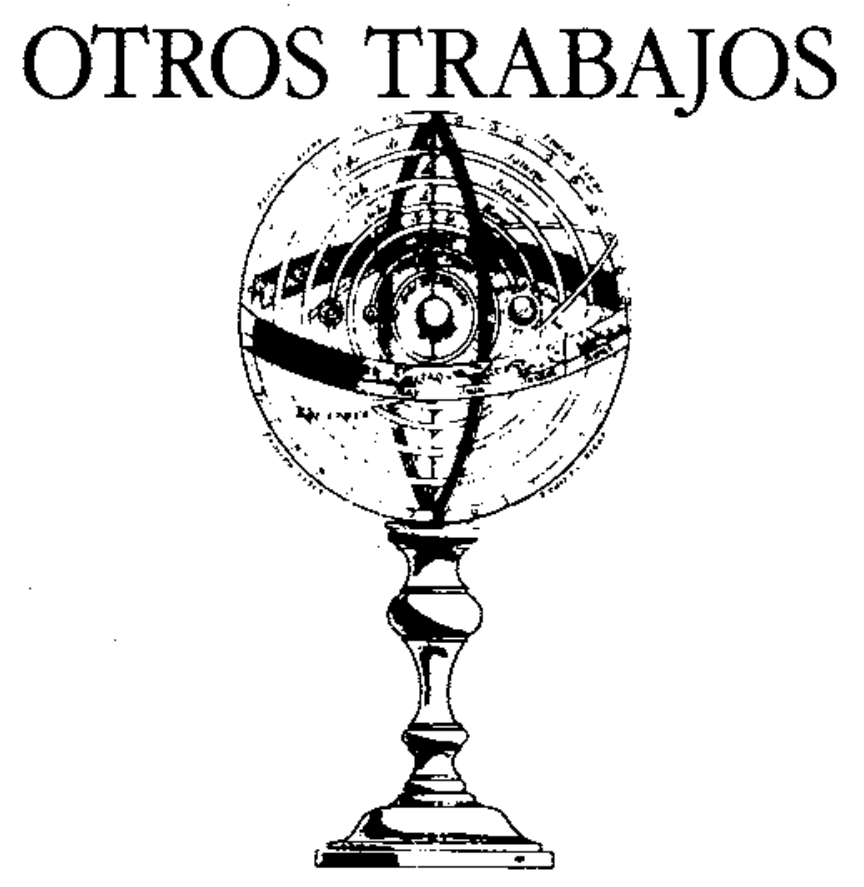

\title{
INTRODUCCIÓN A LA QUÍMICA ORGÁNICA: UN ENFOQUE CURRICULAR
}

SEOANE PRADO, $\mathrm{C}$.

Departamento de Química Orgánica. Facultad de Química. Universidad Complutense. 28040 Madrid.

\section{SUMMARY}

This article examines several alternative approaches to a teaching curriculum in Organic Chemistry at the University level. A special attention is devoted to basic, general concepts and their organization in the introductory topics.

«-Por dónde debo comenzar?, preguntó el Conejo Blanco.

-La respuesta es sencilia, dijo el Rey: Comienza por el principio, continúa hasta llegar al final y entonces para.» Lewis Carrol, Alicia en el País de las Maravillas, 1865.

¿Cuál es el principio de la Química Orgánica? ¿Por dónde comenzar la enseñanza de una ciencia de naturaleza «cíclica»? Para Gutsche y Pasto (1975), no existe un purto de arranque inequívocamente adecuado para la enseñanza de una disciplina en la que « para conocer un tema $\mathrm{A}$ es necesario saber otro, $\mathrm{B}$, cuyo conocimiento exige el de un tercero, $C$, para cuya comprensión es necesaria la de A».

A pesar de ello como, para toda transmisión de conocimientos, es inevitable la adopción de un criterio de orderación, de una cronología expositiva de la Química 
Orgánica, Ia íntima interconexión entre sus distintas materias y los diferentes enfoques posibles hacen difícil la decisión. Básicamente, sin embargo, caben dos planteamientos radicalmente distintos. El primero de ellos se basa en un estudio de los diferentes tipos de reacción que pueden tener lugar sobre los compuestos orgánicos, estructurado sobre consideraciones mecanísticas. Ia segunda posibilidad para la estructuración didáctica de Ia Química Orgánica consiste en aceptar una ordenación por grupos funcionates y realizar un estudio sistemático de cada grupo o familia de compuestos, incorporando en este contexto las reacciones y mecanismos propios de cada grupo funcional.

La primera alternativa presenta la ventaja de una más perfecta racionalización y su tinte teórico parece confetirle mayor altura científica. Además, pucde implicar una menor extensión, ya que el número de tipos de reacción fundamenfales en Química Orgánica es relativamente reducido y cada uno de ellos es aplicable a más de un grupo funcional.

No obstante, la experiencia docente indica que el alumno que principia su estudio de la Química Orgánica asimila mejor la ordenación por funciones, en la que se van incorporando las interpretaciones mecanísticas pertinentes. Elto permite una introducción más pausada y progresiva de la materia, aumentando poco a poco la complejidad de los conceptos que se van enseñando, sin perder por ello la visión de conjunto de las reacciones orgánicas.

La ordenación mecanística, aunque de indudable atractivo para el químico orgánico, exige un conocimiento previo de la disciplina para que de clla pueda sacarse adecuado fruto. No se adapta bien a la crapa formativa en que se encuentra el alumno principiante y es más apropiada para cursos avanzados, en los que el alumno dispone ya de un esquema básico de la Química Orgánica y es capaz de establecer conexiones entre procesos cuya utilidad y alcance ya conoce. Por otra parte, la ordenación por grupos funcionales permite un mejor desarrollo de los aspectos descriptivos de cada familia de compuestos, que, en definitiva, constituyen la verdad última de una ciencia natural como la Química: los hechos.

Desde un punto de vista metodológico, el tratamiento mecanístico de la Química Orgánica deriva de una interpretación apriorística de los hechos experimentales, cuya explicación parece deducirse de una teoría previamente formulada ante el alumno. Sabemos bien que la realidad de una ciencia natural es otra. Las leyes, las interpretaciones, las teorías, se alcanzan por un proceso de inducción, que conduce a una racionalización a posteriori de hechos experimentales previamente conocidos. Alguien tan poco sospechoso de empirismo como Linus Pauling (1980) ha advertido sobre los riesgos de una excesiva teorización. Es preciso evitar que la teoría oculte los conocimientos factuales, en un enfrentamiento entre la «hysicat Chemistry» y la «Chemical Chemistry», como se ha escrito recientemente. Los principios teoricos servirán de soporte al contenido docente, pero sin impedir el estudio de hechos y propiedades. Aunque no es posible, ni aconsejable, un método didáctico estricta- mente histórico-inductivo, ef mejor compromiso es, a nuestro entender, aquél que racionalice cuanto sea posible pero no imprima en el alumno la idca de que la Química Orgánica es una ciencia deductiva.

La ejecución de este planteamicnto exige dos fases didácticas bien diferenciadas: una parte introductoria, para sentar Jos conceptos básicos -a la que nos referiremos con detalle-, y una segunda parte con el estudio sistemático de los compuestos orgánicos. Es la primera parte la que tiene una vital importancia.

El primer contacto con la Química Orgánica tiene como propósito centrar su problemática y su contenido como ciencia, para ofrecer al alumno una perspectiva previa y general de los estudios que comienza. Es necesario encuadrar la Química Orgánica dentro de la Química y poner de manifiesto su relación con la naturaleza viva. Pero es conveniente también situar la Química Orgánica en una perspectiva científica amplia e inciuso en su dimensión social y humana. Clapp (1984) elabora algunas ideas de interés en esta línea.

Previo también al conocimiento detallado de los compuestos orgánicos es el problema de su aislamiento y purificación, algo que ha de darse por sentado en el mancjo real de reacciones y que el alumno no llega, con frecuencia, a captar con plenitud: là necesidad de disponer de compuestos puros si ha de hacerse con ellos una química inteligible.

Conocido el modo de disponer de sustancias puras, se plantea a continuación el problema de las estructuras moleculares y su determinación.

Aunque no es posible, ni se pretende, que el alumno adquiera aquí un completo conocimiento de las técnicas químicas y espectroscópicas de determinación estructural, es preciso que tome temprana conciencia de su necesidad (Arnad 1974). De cste modo, los ejemplos indicados a lo largo de las explicaciones permitirán que cl alumno adquiera la idea de que, sin menosprecio de tos procedimientos químicos, la mayoría de los problemás estructurales se resuelven hoy por métodos espectroscópicos, cuyo conocimiento es esencial para el trabajo en Química Orgánica. Más aún: apuntemos, como nota para el docente, Ia tendencia que empieza a observarse hacia el desarrollo de procedimientos informáticos para la elucidación estructural automatizada, de futuro tan incierto como sugerente (Offermann 1986).

Deben revisarse a continuación una seric de conceptos generales: concepto de radical, grupo funcional y seric homóloga. El concepto de isomería permite enlazar, como resultado del enorme número de estructuras que de ella resultan, con la necesidad de una nomencilatura sistemática, cuya importancia inexcusable se hate asf́ bien patente. El propósito que se persigue es exponer aquí las bases de la nomenclatura sistemática . IUPAC... de los hidrocarburos para apoyar en ella la nonenclatura de las restantes series homólogas. Se dota así al alumno de un lenguaje inicial, «el vocabulario oficial» del idioma que empieza a hablar. Los "vocablos irregulares" . los nombres vulgares *.. habrán de ser introductioios gradualmente. Aún a riesgo de producir alguna confusión en el principiante, no puede ocultarse la desafortunada, 
pero real, falta de universalidad en el empleo de la nomenclatura sistemática. Junto a los nombres triviales, en ocasiones de ubicua aplicación; dos fuentes bibliográficas de la trascendencia del Belstein y el Chemical Abstracts emplean sistemas de nomenclatura que no coinciden ni entre sí ni con el preconizado por la IUPAC en aspectos importantes.

Aunque claramente prematuro para fines didácticos, mencionaremos la existencia de intentos de reforma radical de la nomenclatura orgánica. Lozac'h (1979) ha propuesto la «nomenclatura nodal», basada cn el esqueleto atómico de la molécula y su sistema reticular de enlace, prescindiendo, en principio, de la nataraleza de los tomos y tipos de enlace. Se logra así un nombre bise, que luego se completa con esos aspectos. Más recientemente, Hirayama (1984) ha recogido los planteamientos generales del sistema Hirn de nomenclatura. Es un sistema muy simple y lógico basado en un centenar de palabras básicas que permite una total correspondencia biunívoca, sin ambigüedades, entre nombre y estructura. El resultado es una notación lineal, tan cómodamente utilizable por el químico como por sus ordenadores, 10 que tal vez convierta este sistema en el ideal cuando es precisa esta interacción. Fin efecto, la antigua informatización de estructuras exigía el uso de notaciones e índices de difícil comprensión para el no iniciado y sin aplicación fuera del campo informático, como ocurre con la «Wiswesser Line Notation» (Gibson y Granito 1972). Resulta muy difícil para el químico orgánico reconocer al naftaleno como $\mathrm{L} 66 \mathrm{~J}$, su notación Wiswesser (Bonchev 1983).

Un último aspecto de interés sobre nomenclatura st: refiere a la estandarización en lengua castellana de lá! ortografía y pronunciación de los términos químicos, un problema que los anglosajones se plantearon hace ya ticmpo (American Chemical Society 1934) y a cuya clarificación en nuestro país puede contribuir la publicación del Diccionario de Términos Científicos de la Real Academia de Ciencias.

El estudio de la nomenclatura resulta sicmpre árido y es preciso hacer comprender al alumno la imperiosa necesidad de disponer de este lenguaje. Hace ya un siglo y medio, Scoffem (1848) alentaba a sus alumnos frente al esfuerzo de aprender nomenclatura con palabras difíciles de superar:

«Recuerden que un niño no disfruta aprendiendo el alfabeto; pero sería, ciertamente, una lástima que se viese privado de todas las riquezas de la literatura y de la ciencia por la sola molestia de aprender las letras.”

El punto siguiente lo ocupa una revisión de las ideas fundamentales sobre orbitales y enlaces en Química Orgánica. Ha de prestarse atención a la formulación de estructuras de Iewis que, un tanto menospreciadas en cursos de Química General, han de manejarse con una soltura que no siempre tiene el alumno que comienza a estudiar Química Orgánica (Zandler y Tolaty 1984, Bradley y Gewans 1985a).

Los desplazamientos electrónicos en moléculas orgánicas (Sacher 1983) son un aspecto cuyas bases habrán de establecerse también en este momento, al igual que ocurre con los conceptos de hibridación y geometría molecular (Kikuchi y Suzuki 1985). El uso de modelos de los distintos híbridos, bien comerciales (Gallenkamp) o «caseros» (Roberts y Traynham 1976) es de utilidad para ayudar a fijar la imagen geométrica en el alumno. Una advertencia debe hacerse respecto a este problema, que tiene incluso alcance general. La teorización con que normalmente se plantea la explicación del enlace hace que el alumno pierda a veces la noción de la diferencia que existe entre un modelo o tcoría de enlace y la realidad física que le dio origen. ¿Sabrían todos los alumnos matizar la diferencia entre estas dos afirmaciones?:

-El metano es una molécula tetraédrica porque el carbono tiene hibridación $\mathrm{sp}^{3}$.

-Puesto que el metano es una molécula tetraédrica, la hibridación del carbono es $\mathrm{sp}^{3}$.

Las fuerzas intermoleculares completan esta temática. Es preciso evitar que el alumno menosprecie su importancia, haciéndole ver la trascendencia que tienen al determinar las propiedades fisicoquímicas de las molé. culas orgánicas, especialmente en el ámbito biológico (Frieden 1975).

El conocimiento de la estructura electrónica de las moléculas orgánicas que así se acaba de adquirir abre el camino para la consideración general de las reacciones orgánicas (Bradley y Gewans 1985) y su sistemática y clasificación por tipos.

Con fines docentes, se mantiene la clasificación tradi. cional de las reacciones orgánicas. De cara al profesor, sin embargo, puede anotarse que, desde hace algún tiempo, vienen presentándose, a veces por firmas muy autorizadas (Hendrickson 1978, Roberts 1978), nuevas propuestas de clasificación y nomenclatura dc las reac. ciones orgánicas, un tema del que la IUPAC está también ocupándose.

Ln este contexto, el sistema de símbolos de reacción que viene utilizando el «Theilheimer» (Theilheimer's Synthetic Methods of Organic Chemistry) tiene un carácter pionero como intento de racionalización. Scgún, este sistema, sc clasifican las reacciones de acuerdo con : a) cl enlace que se forma, $b$ ) el tipo de transformación, $c$ ) el enlace que se rompe o cl elemento que se climina. A cada uno de estos conceptos se asignan unos símbolos que, en grupos de tres, describen una reacción. El primer símboto indica los elementos que se unen en el nuevo eniace. El segundo símbolo designa uno de los cuatro tipos de transformación posible: adición $(\downarrow)$, transposición $(\bigcap)$, intercambio $(\uparrow \downarrow$ y eliminación $(\uparrow)$. El último símbolo indica los elementos que estaban unidos en el enlace y que la reacción destruye o, en el caso de climinaciones, el elemento que se elimina. Algún ejemplo puede resultar ilustrativo:

$$
\begin{array}{ll}
\mathrm{R}-\mathrm{CH}=\mathrm{CH}_{2} \stackrel{\mathrm{H}_{2} \mathrm{O}}{\longrightarrow} \mathrm{R}-\mathrm{CH}-\mathrm{CH}_{3} & \mathrm{OC} \Downarrow \mathrm{CC} \\
\mathrm{R}-\mathrm{Cl} \stackrel{\mathrm{CN}}{\longrightarrow} \mathrm{R}-\mathrm{CN} & \mathrm{CC} \Uparrow \mathrm{Ha} !
\end{array}
$$


Una explicación más amplia puede encontrarse en el trabajo de Finch y Mitchel (1984). En la presentación general de las reacciones orgánicas ha de incluirse una consideración elemental del concepto de mecanismo de reacción en relación con los parámetros termodinámicos -entalpía, cntropía, energía libre- (Hamori 1975) que permiten comparar y diferenciar la espontaneidad y la velocidad de una reacción orgánica, to que permite llevar al alumno a percibir la importancia de los aspectos cientificos.

El estudio se completa con una discusión de los diferentes intermedios de reacción. El carácter transitorio de las partículas intermedias, sus tiempos de vida y el rango de sus estabilidades en relación con moléculas estables presenta una problemática no siempre bien captada por el alumno. La comparación de los intermedios transitorios (radicales, carbocationes) con ejemplos estables de tales especies, como el radical trifenilmetilo, puede ser una ayuda, si bien es cierto que la generación de carbocationes sencillos estables en condiciones adecuadas, posible desde los primeros trabajos de Olah (1967), viene a complicar aún más la situación para el docente. Por otro lado, los cationes vinilo se tratan habitualmente como especies extremadamente inestables y de difícil formación, incluso como intermedios transitorios. No obstante, los trabajos de Subramanian y Hanack (1975), han puesto de manifiesto la facilidad de formación de ciertos cationes vinilo por rotura heterolítica en solvólisis de derivados vinílicos con grupos salientes muy buenos (triflatos). Se trata de un hecho importante que algunos textos generales empiezan ya a incorporar. Es importante también hacer ver al alumno la diferencia entre la generación de radicales y carbocationes, posible sobre esqueletos alquilo sencillos, y la necesidad, en el caso de carbaniones, de grupos que estabilizan la carga negativa. Se sosiaya así el riesgo, tan frecuente, de que el estudiante considere la formación de carbaniones alquilo y pretenda manejarlos, como a veces ocurre, de modo equivalente a los carbocationes o radicales. La mención de los recientes trabajos de von Schleyer (1986) y el rotundo título de su artículo pueden refor $z a r$ esta idea: "The Ethyl, 1- and 2- Propyl and Other Simple Alkyl Carbanions do not Exist».

El conjunto de conceptos que hasta aquí se habrán adquirido posibilitará el estudio detenido que, de cada mecanismo, se hará en el punto del programa en que corresponda, en relación con el tipo de compuestos orgánicos en que se produce.

Conectamos así con la segunda parte de un curso de
Química Orgánica: el estudio sistemático de las distintas series de compuestos, sobre lo que haremos tan sólo un comentario general.

La estructuración propuesta se basa en una ordenación por grupos funcionales, pero entretejida con las bases mecanísticas que dan unidad y coherencia lógica a la Química Orgánica. En cada tipo de compuestos se estudian tres aspectos fundamentales interdependientes entre sí: la estructura, el comportamiento químico y los métodos de síntesis. El criterio a seguir se basa en desarroltar la primera, para fundamentar en ella el comportamiento químico que ha de tratarse en profundidad, conexplicación de las reacciones importantes y su racionalización en función de la estructura. Los métodos de preparación. que no son, en definitiva, más que las reacciones propias de otros compuestos, pueden tratarse más abreviadamente insistiendo, más que en aspectos mecanísticos, en las facetas prácticas de la síntesis. Deben también indicarse, comparándolas, las preparaciones de laboratorio y los métodos de preparación de carácter industrial.

Una solución alternativa consiste en separar el estudio de la estructura del de la reactividad, en la línea adoptada por la obra clásica de Allinger. No obstante, el estudio de la reactividad de cada familia de compuestos inmediatamente después de la estructura de su grupo funcional permite una más directa correlación entre cl comportamiento químico y su base estructural.

Es evidente que un esquema como el propuesto implica que la mayoría de las reacciones orgánicas han de ser tratadas dos veces: como reacción de un compuesto y como síntesis de otro. Este hecho no deja de ser didácticamente positivo y, por otra parte, permite estudiar cada reacción desde dos puntos de vista diferentes: la relación estructura-reactividad, en un caso, y las aplicaciones y limitaciones sintéticas en el otro.

Una última observación: en principio, y teniendo en cuenta la naturaleza «cíclica» de la Química Orgánica a que hemos aludido, será posible comenzar su estudio por cualquiera de sus capítulos. No obstante, creemos aconsejable, en la presentación de los distintos grupos funcionales, adoptar una secuencia creciente de complejidad estructural, de modo que los grupos funcionales con enlaces múltiples se posponen a los que contienen solamente eniaces sencillos. En efecto, ia mayor complejidad en la reactividad de los grupos funcionales con enlaces puede ser mejor comprendida por el alumno tras haber estudiado la reactividad de los grupos funcionales con enlaces sencillos. 


\section{REFERENCIAS BIBLIOGRÁFICAS}

AMERICAN CHEMICAL SOCIETY, 1934. The Proninciation of Chemical Words. A Report on the Nomenclature, Spelling and Pronunciation Commitee of the American Chemical Society, Industrial and Engineering Chemistry., $12(10)$, p. 202.

ARNAD, C., 1974. Structural Determination in Organic Chemistry, Journal of Chemical Education, 51, a. 819.

BONCHEV, D., 1983. Information Indices for Characterization of Chemical Structures. (Wiley).

BRADLEY, J. y GEWANS, G.C., 1985a. Lewis and the Electron-Pair Bond, Education in Chemistry, 22, p. 48.

BRADLEY, J.D. y GEWANS, G.C., 1985. Teaching and Learning About Organic Chemical Reactions, Education in Chemistry, 22, p. 74 .

CLAPP, L.B., 1984. Why Teach Organic Chemistry?, Joxrnal of Chemical Education, 6I, p. 239.

FINCH, A.F. y MITCHEL, P.R., 1984. Getting the Best out of Theilheimer's Synthetic Methods of Organic Chemistry. (Karger).

FRIEDEN, E., 1975. Non-covalent Interations: Key to Biological Flexibility and Specifity, Journal of Chemical Educction, 52, p. 755.

GIBSON, G.W. y GRANITO, Ch., 1972. Wiswesser Line Notation, American Laboratory, $4,27$.

GUTSCHE, C.D. y PASTO, D.J., 1975. Fundamentals of Organic Chemistry. (Prentice Hall).

HAMORI, E., 1975. Ilustration of free Energy Changes in Chemical Reactions, Journal of Chemical Education, 52 , p. 371.

HENDRICKSON, J.B., 1978. A Logical Characterization of Organic Chemistry, Journal of Chemical Education, 54, p. 216.
HIRAYAMA, K., 1984. The Hirn System: Nomenclature of Organic Chemistry Principles. (Springer-Verlag).

KIKUCHI, O. y SUZUKI, K., 1985, Orbital Shape Representations, Journal of Chemical Education, 62, p. 206.

LOZAC'H, N., GOODSON, A.L. y POWELL, W., 1979. Nodal Nomenclature. General Principles, Angewandte Chemie, International Edition in English., 18, p. 884.

OFFERMANN, W. et al., 1986. Towards Automated Structure Elucidation, Tetrahedron, 42 , p. 2215.

OLAH, G. et al., 1967. Stable Carbonium Ions. Protonated Aliphatic Alcohols and Their Cleavage to Carbonium Ions, Journal of the American Chemical Society., 89, p. 3576.

PAULING, L., 1980. Prospects and Retrospects in Chemical Education, Journal of Chemical Education, 57, p. 39.

ROBERTS, D.C., 1978. Journal of Organic Chemistry., 43, p. 1473

ROBERTS, R. y TRAYNHAM, J., 1976. Molecular Geometry: As Easy as Blowing Up Ballons, Journal of Chemical Education, 53, p. 233.

SACHER, E., 1983. Tetrahedron Letters, 27, p. 4683.

SCHLEYER, P.v.R., 1986. Tetrahedron Letters, , 27, p. 4411.

SCOFFERN, I., 1848, Chemistry, No Mistery or a Lecturer's Bequest. $2^{\underline{a}}$ ed. (A. Hall: London).

SUBRAMANIAN, L. y HANACK, M., 1975. Generation of Vinyl Cations by Solvolysis Reaction: A Lesser Known Genre of Carbenium Ions, Journal of Chemical Education, 52, p. 80 .

ZANDLER, M. y TOLATY, E., 1984. The 6N+2 Rule for Writing Lewis Octet, Structures, Journal of Chemical Education, 61, p. 124 . 